DOI: $10.1002 /(($ please add manuscript number $))$

Article type: Communication

\title{
Giant enhancement in the supercapacitance of NiFe-graphene nanocomposites induced by a magnetic field.
}

By Jorge Romero, Helena Prima-Garcia, Maria Varela, Sara G. Miralles, Victor Oestreicher, Gonzalo Abellán* and Eugenio Coronado*.

J. Romero, Dr. H. Prima-Garcia, Dr. S. G. Miralles, Dr. V. Oestreicher, Dr. G. Abellán, Prof. E. Coronado

Instituto de Ciencia Molecular (ICMol), Universidad de Valencia. Catedrático José Beltrán 2, 46890 Paterna, Spain.

Dr. M. Valera

Universidad Complutense de Madrid, Instituto Pluridisciplinar, Instituto de Magnetismo Aplicado \& Departamento de Física de Materiales, Madrid 28040, Spain.

Dr. G. Abellán

Department of Chemistry and Pharmacy and Joint Institute of Advanced Materials and Processes (ZMP)

Friedrich-Alexander-Universität Erlangen-Nürnberg (FAU), Nikolaus Fiebiger-Strasse 10, 91058 Erlangen and Dr.-Mack Strasse 81, 90762 Fürth, (Germany).

E-mail: gonzalo.abellan@uv.es (G.A.) eugenio.coronado@uv.es (E.C.)

Keywords: Supercapacitors, magnetic, metal-segregation, nanocomposite, microscopy.

The rapid rise in energy demand of the last years has prompted the search of low-cost alternatives for energy storage, being supercapacitors (SCs) one of the most important devices. Here we show that a dramatic enhancement ( $c a .1200 \%$, from 145 to $1850 \mathrm{~F} \cdot \mathrm{g}^{-1}$ ) of the specific capacitance of a hybrid stimuli-responsive $\mathrm{FeNi}_{3}$-graphene electrode material can be achieved when the charge/discharge 20 cycling is performed in presence of an applied magnetic field of $4000 \mathrm{G}$. This result is related with an unprecedented magnetic-field induced metal segregation of the $\mathrm{FeNi}_{3}$ nanoparticles during the cycling, which results in the appearance of small Ni clusters $(<5 \mathrm{~nm})$ and, consequently, in an increase of pseudocapacitive sites. Our results open the door to a systematic improvement of the capacitance values in hybrid supercapacitors, while moving the research in 25 this area towards the development of magnetically-addressable energy storage devices. 
Due to the pressing increase of global warming effects and the finite nature of fossil fuels, the search for alternative energy sources has dramatically boosted during the last few years. ${ }^{[1,2]}$ Hybrid systems consisting of layered materials have taken the lead in the design of advanced nanomaterials based on their superior electrochemical performance reliant on their unique physical properties, control over their organization and efficient electronic communication due to hybridization between components. ${ }^{[3,4]}$ Energy-related applications that could benefit the most from these hybrid systems include flexible electronics, lithium and sodium ion batteries with efficient anodes and cathodes, solar cells or supercapacitors with high energy and power densities, useful for industrial stationary applications, automotive transportation and portable electronic applications. ${ }^{[5]}$ Such supercapacitors (SCs) are key elements among energy storage devices due to their potential to deliver high power densities in short periods of time with high cyclability and durability. ${ }^{[6]}$ In supercapacitive devices, recent efforts trying to improve the energy and power densities have been devoted to optimize the internal configuration of the capacitor. This includes new synthetic approaches for electrode materials, novel capacitor configurations, or tailored porous hierarchies. Even though the fast development of electrode materials for SCs has dramatically increased the performance of these devices, there is still room for further improvement. ${ }^{[7]}$ In this context, a loosely investigated approach consists in the application of an external stimulus, such as a magnetic field. ${ }^{\left[{ }^{8-10]}\right.}$ In principle, magnetic fields may affect the capacitive internal resistances since they can induce complex magnetohydrodynamic (MHD) phenomena in the electrolytes, offering a new tool for tuning the performance of SCs. Despite some remarkable efforts, this effect remains very small. ${ }^{[11,12]}$

Here, we present an approach to overcome this limitation, consisting of incorporating magnetic nanoparticles in the electrochemically active material, which can be easily magnetized by an external field. We have recently reported the development of a low- 
temperature route to the synthesis of hierarchically structured magnetic graphene- $\mathrm{FeNi}_{3}$ nanocomposites (MNC) using hybrid layered double hydroxides nanoreactors as precursors. ${ }^{[13,14]}$ These materials exhibit excellent performances as supercapacitors, and at the same time they behave as soft magnets at room temperature. ${ }^{[14,15]}$ Herein we demonstrate that the application of a relatively weak magnetic field of $4000 \mathrm{G}$ during galvanostatic chargedischarge cycles results in a dramatic enhancement of the specific capacitance near $850 \%$. A thorough characterization by means of electron microscopies, magnetic properties, magneto transport, x-ray photoelectron spectroscopy (XPS), Raman spectroscopy and electrochemical measurements (cyclic voltammetry, galvanostatic charge/discharge and potentiostatic electrochemical impedance spectroscopy, PEIS) revealed that the origin of this groundbreaking finding is a full metal segregation of the magnetic nanoparticles induced by the magnetic field.

To evaluate the performance of the pristine MNC under an external applied magnetic field we used a home-made three electrode electrochemical cell fixed between the two magnetic poles of a controllable electromagnet and $\mathrm{KOH} 6 \mathrm{M}$ as electrolyte (Figure 1 and supplementary materials Figure SI 1). The cyclic voltammograms under a magnetic field present an enlarged current and enclosed area of the curve, in which the anodic and cathodic peaks showed a shift towards more positive and negative potentials, respectively, indicative of a higher electrochemical reaction activity (Figure SI 2-3). In the studied potential window, the main contribution to the electrochemical properties arises from the formation of a $\mathrm{NiO}$ shell. Concretely, a reversible process of insertion and extraction of $\mathrm{OH}^{-}$ions should be expected, following the reaction: $\mathrm{Ni}^{\mathrm{iI}} \mathrm{O}+\mathrm{OH}^{-} \leftrightarrow \mathrm{Ni}^{\mathrm{III}} \mathrm{OOH}+\mathrm{e}^{-} \cdot{ }^{[4]}$ Galvanostatic charge-discharge cycles without applying a magnetic field have been studied (MNC-C) showing an increase of the capacitance with the number of cycles of $c a .140 \%$ after 300 cycles (from $155 \mathrm{~F} \cdot \mathrm{g}^{-1}$ with a current density of $1 \mathrm{~A} \cdot \mathrm{g}^{-1}$ to $400 \mathrm{~F} \cdot \mathrm{g}^{-1}$ ) (Figure SI 4). In this sort of materials, the capacitance 
retention increases as a consequence of the activation of the $\mathrm{MNC}$, usually generating metal oxides. ${ }^{[11,12,16]}$ Control experiments show that the performances are lower for the separate components of the nanocomposite ( $\mathrm{FeNi}_{3} \mathrm{NPs}$ and graphene), indicative of a synergistic effect of the two components in the MNC (Figure SI 4). In turn, the application of a magnetic field during the galvanostatic charge-discharge cycles (MNC-MC) manifests a more drastic increase of the capacitance retention, whether the field is applied constantly or gradually (Figure SI 5). The best performance was obtained for the highest studied field, that is at a constant applied field of $4000 \mathrm{G}$ (where the MNC is fully magnetized, as we can see in Figure SI 6), obtaining an increase of $c a .600 \%$ in the specific capacitance after 300 cycles (from $155 \mathrm{~F} \cdot \mathrm{g}^{-1}$ to $\left.1100 \mathrm{~F} \cdot \mathrm{g}^{-1}\right)$. This specific capacitance increases even more and tends to a leveling after 1000 cycles, reaching a capacity of $c a .1850 \pm 180 \mathrm{~F} \cdot \mathrm{g}^{-1}$ (Figure 1.b). This represents an improvement of $c a .1240 \%$, increasing $1140 \%$ with respect to the initial value, in contrast to the $270 \%\left(550 \pm 60 \mathrm{~F} \cdot \mathrm{g}^{-1}\right)$ of the MNC-C sample. As we can see in this Figure, the performance in the capacitance is significantly higher when the material undergoes charge/discharge cycles in presence of a magnetic field. Also, we performed a comparative study of different densities of active material in the electrode, showing a better capacitance increase with $0.3 \mathrm{mg} \cdot \mathrm{cm}^{-2}$ (see Figure SI 9). Furthermore, the MNC-MC material shows a good stability with capacitance retention greater than $90 \%$ after 10,000 cycles (Figure SI 12). This is, to the best of our knowledge, the highest improvement ever obtained in the specific capacitance by means of magnetic fields. We should notice that the only precedent in this context has been reported by Guo and co-workers in a system formed by microtubular cotton fabrics decorated with metal nanoparticles. ${ }^{[12]}$ In this work the magnetic field effect has produced an increase in the capacitance of a $70 \%$ with values of $60 \mathrm{~F} \cdot \mathrm{g}^{-1}$ and a poor cyclability of less than 800 cycles. 
For better understanding the fundamental behaviour of the magnetocapacitive $\mathrm{FeNi}_{3}$-carbon nanocomposites, PEIS measurements were performed in the MNC pristine sample with and without an externally applied magnetic field (see Methods and Figure SI 13 for a detailed description). Thus, three internal resistances are affected by the magnetic field: the solution resistance in bulk electrolyte $\left(\mathrm{R}_{\mathrm{S}}\right)$, the electrode/electrolyte interfacial charge transfer resistance $\left(\mathrm{R}_{\mathrm{CT}}\right)$ directly related to the electrode's resistance, and the low frequency leakage resistance $\left(\mathrm{R}_{\text {leak }}\right)$ flowing across the double layer zone at the electrode-electrolyte interface. The most pronounced effect was observed for the $R_{\text {leak, }}$ increasing from $764 \Omega$ to $920 \Omega$ with the applied magnetic field, indicating that $R_{\text {leak }}$ is responsible for the improvement in the energy storage performance of our device, and suggesting that the in presence of a magnetic field the electrolytes can foster the development of the oxide surface, increasing notably the resistance of the double layer. ${ }^{[11]}$

Statistical Raman spectroscopy (SRS) has been used to characterize the degree of graphitization and homogeneity of the materials. ${ }^{[17]}$ The mean spectra of MNC (average of an area of $20 \times 20 \mu \mathrm{m}^{2}$ ), measured in backscattering geometry and using a $533 \mathrm{~nm}$ excitation wavelength, revealed the characteristic bands of graphene (i.e. D, G and 2D) showing an average $\mathrm{I}_{\mathrm{D}} / \mathrm{I}_{\mathrm{G}}$ ratio of 1.2 and 1.1 before and after the application of the magnetic field, respectively (Figure 2a). This suggests a slight decrease in the functionalization of the graphene matrix and indicates that it is resilient to the cycling process. The presence of the encapsulated metal oxides was detected after increasing one order of magnitude the laser power to $1.8 \mathrm{~mW}$, with characteristic signals of $\mathrm{M}-\mathrm{O}$ species in the $400-800 \mathrm{~cm}^{-1}$ region, most likely $\mathrm{NiO}$ and/or $\mathrm{FeO}_{\mathrm{x}}$ (see inset in Figure 2a). The MNC-MC exhibited a more pronounced fluorescence background than that of the pristine counterpart. In stark contrast, the sample submitted to cycles without any magnetic field (MNC-C) exhibited a much more laser-sensitive carbon matrix, with an increased fluorescence background, indicative of the 
presence of metallic species and/or amorphous carbon indeed, a $633 \mathrm{~nm}$ excitation wavelength was required to record the average spectra (see Figure SI 14).

In order to shed light into the surface oxidation of the materials, X-ray photoelectron spectroscopy (XPS) was performed. Each of the two spin-orbit components of the Ni $2 p$ spectra shows three resolved features; those located at 853.2 and $856.4 \mathrm{eV}$ can be attributed to metallic and divalent $\mathrm{Ni}$ (mainly $\mathrm{NiO}$ and $\mathrm{Ni}(\mathrm{OH})_{2}$ ), respectively, whereas the one located at $862.5 \mathrm{eV}$ can be attributed to the so-called $6 \mathrm{eV}$ Ni satellite. ${ }^{[15]}$ Compared to pristine $\mathrm{MNC}$, both MNC-C and MNC-MC show the disappearance of the Ni metal signal at $853.2 \mathrm{eV}$, indicative of surface oxidation. Remarkably, a more pronounced $\mathrm{Ni}-\mathrm{O}$ peak is present in the MNC-MC. Similarly, the Fe 2p survey revealed photoelectron peaks at around 707.7, 711 and $725 \mathrm{eV}$ in the pristine material, confirming the presence of zero-valent iron (Fe 2p3/2), which completely vanishes in the cycled samples (see Figure $2 \mathrm{c}-\mathrm{d}$ and supporting information Figure SI 15-21). The application of the magnetic field induces a decrease in the $\mathrm{C}-\mathrm{O}$ contribution of the $\mathrm{C} 1 \mathrm{~s}$ high resolution spectra, which explains the reduced $\mathrm{I}_{\mathrm{D}} / \mathrm{I}_{\mathrm{G}}$ ratio previously observed in Raman spectroscopy.

Further insights on the chemical structural evolution were obtained from magnetic measurements at $300 \mathrm{~K}$. MNC behave as soft magnet showing small coercive fields, and being fully magnetized at low fields at room temperature. ${ }^{[14]}$ The magnetic field exerts a big influence on the chemical reactions occurring in the MNC upon cycling. Thus, the hysteresis loops of the different materials showed a decrease of $c a .58 \%$ in the saturation magnetization $\left(\mathrm{M}_{\mathrm{s}}\right)$, from $\mathrm{ca} .88$ to $51 \mathrm{emu} \cdot \mathrm{g}^{-1}$ in the MNC-MC. Moreover, no significant differences in both remnant magnetization $\left(\mathrm{M}_{\mathrm{r}}\right)$, and coercivity $\left(\mathrm{H}_{\mathrm{c}}\right)$ values were measured (Figure SI 6). Furthermore, conductivity measurements show an increase in the resistance of MNC-MC of two orders of magnitude compared to the $\operatorname{MNC}\left(6.54 \mathrm{k} \Omega \cdot \mathrm{cm}^{-1}\right.$ to $200 \Omega \cdot \mathrm{cm}^{-1}$, respectively), shifting from a metal conductor behavior to a semiconductor (Figure SI 22-24). Overall, EIS, 
XPS, the magnetic measurements, and the increase in the resistance confirm that the oxidation processes at the surface of the metal nanoparticles are enhanced by the magnetic field.

However, although all these techniques point towards a preferential growth of electroactive oxide layers and a better structural resilience of the graphene matrix when the charge/discharge cycles take place in presence of a magnetic field, a direct microscopic evidence of this effect is strongly required. To tackle this challenge, we analyzed MNC, MNC-C and MNC-MC samples by real-space techniques sensitive to both chemistry and structure, such as atomic-resolution aberration-corrected scanning transmission electron microscopy and electron energy-loss spectroscopy (STEM-EELS) at both 80 and $200 \mathrm{kV}$. Low magnification images of the pristine MNC material measured at $200 \mathrm{kV}$ show a distribution of particles with uneven shapes and sizes in the $5-200 \mathrm{~nm}$ range, as depicted in the low magnification annular bright field (ABF) image in Figure 3a. The particles are coated with a $\mathrm{C}$ layer, as observed in the atomic resolution $\mathrm{ABF}$ images. The nanoparticles are crystalline but extended defects such as stacking faults and twin boundaries are often observed (even in the smaller particles, in the few nm size range). Observations at $80 \mathrm{kV}$ allow minimizing electron beam irradiation damage. EEL spectrum imaging was used to obtain chemical maps of the samples. The particle composition was quite even, approximately $(74 \pm 2) \% \mathrm{Ni} /(26 \pm 2) \% \mathrm{Fe}$ in excellent accordance with the expected theoretical composition of $\mathrm{FeNi}_{3}$. Some surface oxidation was detected, mostly associated with an enhancement of the surface Fe signal, which comes along with a slight increase of the Fe $L_{23}$ intensity ratio. These findings point to a significant surface Fe oxidation (see Figure 3b), in line with previous reports on preferential formation of Fe-oxide on the surface of NiFe-permalloys, associated with the depletion of Fe from the bulk to the surface. ${ }^{[18,19]}$ Indeed, the fine structure of the $\mathrm{O} K$ edge suggests a strong Fe-O like signal (paramagnetic) ${ }^{[20]}$. No major segregation of metals or 
heavier species on the $\mathrm{C}$ matrix was observed. It is worth mentioning that the $\mathrm{C}$ coating was very stable, even for observations carried out at $200 \mathrm{kV}$.

On the other hand, the sample submitted to charge/discharge cycles in absence of a magnetic field (MNC-C) exhibited a quite unstable carbon matrix under the electron beam degrading fast at $200 \mathrm{kV}$, in excellent accordance to Raman spectroscopic studies. Low magnification images exhibit a higher density of small particles, with large amounts of smaller clusters in the few nm size range. Intermediate magnification images acquired at $80 \mathrm{kV}$ to minimize the damage confirmed that a relatively high amount of material segregates on the $\mathrm{C}$ support in the form of small clusters $<5 \mathrm{~nm}$ (see Figure SI 25). This finding indicates that the initial $\mathrm{FeNi}_{3}$ nanoparticles were electrochemically active and broke into small ones during the chargedischarge cycles. EELS maps show again a strong surface Fe oxidation. However, in this case small Ni-rich clusters have segregated outside the particles. The presence of the small clusters suggests activated electrochemical behaviour, in agreement with the observed improved capacitance.

Finally, for the sample submitted to charge/discharge cycles in presence of a magnetic field (MNC-MC), simultaneously acquired $\mathrm{ABF}$ and high angle annular dark field (HAADF) images, revealed the wide formation of small precipitates, along with needle-shaped particles all over the sample (Figure 4a). The smaller particles tend to exhibit a core-shell like structure, single- or mono-domain inside, with high crystallinity, and appeared coated by uneven C layers (see Figure SI 26 for additional characterization). Moreover crystalline needle-shaped clusters and other crystalline small $(<5 \mathrm{~nm})$ particles can be observed on the C matrix. EELS analysis demonstrated that all clusters and needles consist of $\mathrm{Ni}, \mathrm{Fe}$ and $\mathrm{O}$. A detailed analysis shows again a strong oxidation of the surface of the particles, which is mostly Fe oxide. Indeed, EELS fine structure, measured on the particles surface revealed that the intensity of the $\mathrm{O} K$ onset pre-peak increases, pointing to a more oxidized compound ${ }^{26}$. 
However, an important finding was discovered when imaging the small clusters: they exhibit a strong $\mathrm{Ni}-\mathrm{Fe}$ segregation. Figure $4 \mathrm{~b}$ shows a total $\mathrm{Ni}-\mathrm{Fe}$ separation with the small Fe-rich clusters heavily oxidized, while the Ni-rich clusters remain metallic and are only oxidized in the surface.

In order to clarify whether the magnetic-field metal segregation requires an electrochemical process or not, we have performed forefront in-situ STEM-EELS analysis under the magnetic field of the microscope objective lens (of the order of 2T) and temperatures between room temperature (RT) and $400^{\circ} \mathrm{C}$. High resolution images of the pristine MNC sample exhibit cluster free surfaces (Figure 5a, left and 5b, top). However, after 15 hours of exposure to the magnetic field at room temperature (Figure 5a, middle), some surface segregation has occurred in the form of an a noticeable density of nanometric surface clusters. $\mathrm{O} K$, Fe $L_{2,3}$ and Ni $L_{2,3}$ EELS maps (Figure 5b, middle) confirm an inhomogeneous composition (along with oxidation) of the surface and such clusters. This segregation can be significantly sped up via heating. The right panel of Figure 5a, exhibits an image of the same particle, after being annealed in-situ at $400^{\circ} \mathrm{C}$ for approximately 30 minutes and then quenched back to RT. The surface clusters have increased in size, almost duplicating their volume. The corresponding compositional maps in Figure 5b (bottom row) denote an enhanced segregation of $\mathrm{Fe}$ and $\mathrm{Ni}$, along with an inhomogeneous oxidation reminiscent of the core-shell morphology of the MNC-MC samples depicted in Figure 4b. These results point toward a significant temperature and field activated $\mathrm{Fe} / \mathrm{Ni}$ surface segregation mechanism, which can be readily observed in videos acquired during subsequent in-situ annealings at $400^{\circ} \mathrm{C}$, always under magnetic field (see movies in Movie SI 1-2, depicting the particle evolution at high temperatures). Moreover, these show that temperature seems to play a determining key role fostering the Fe migration to the surface and growing of crystalline segregated particles, which grow by coalescence. These results confirm the magnetic field-induced segregation process highlighting the 
importance of providing energy to the system either in the form of thermal agitation or electrochemical potential.

In view of the previous results we can give an explication of the magnetic field effects in the redox processes. According to White and co-workers ${ }^{[21]}$, when a magnetic field is externally imposed in the electrochemical cell, the mass transport of electrolyte at the electrolyte/electrode interface is altered, which changes the electrical double layer at the electrode surface. ${ }^{[22]}$ These changes are mainly attributed to the MHD phenomenon, which accelerates the ion transportation rate within the electrolyte solution and the electron exchange at the electrolyte/electrode interface. ${ }^{[9,23]}$ Moreover, the magnetic field may generate magnetophoretic forces (i.e. Kelvin force and Maxwell stress) acting on generated paramagnetic ions, always present because any redox process where a single electron is transferred necessarily involves paramagnetic species. ${ }^{[10]}$ In fact, depending on the magnetic field uniformity, the Maxwell stress may deform the shape of ion clouds near the electrode surface influencing the capacitance of the electrical double layer. In general, the magnetic field effects remain small for these redox processes. However, in our case, these effects are strongly enhanced by the presence of ferromagnetic $\mathrm{FeNi}_{3}$ nanoparticles which are fully magnetized even in presence of fields as small as $4000 \mathrm{G}$, thanks to their soft ferro- magnetic nature. This leads to an increase in the total magnetic field at the very surface of the nanoparticle due to the generation of stray fields. ${ }^{[24]}$ The fact that minimum improvements in the capacitance retention are observed upon applying higher mag- netic fields may support this hypothesis. As a consequence, an intense local gradient force will be generated at the boundary layer region of every single particle. The magnetic force will govern the dynamics of diamagnetic and paramagnetic molecules moving away from and toward regions of higher magnetic field strength, respectively. Moreover, the presence of magnetic fields could decrease the surface energy of the nanoparticles promoting the formation of new crystalline 
structures, as previously observed for $\mathrm{NiO}, \mathrm{Co}_{3} \mathrm{O}_{4}, \mathrm{NdFeB}$ and $\mathrm{NiCo}_{2} \mathrm{O}_{4}$ nanoparticles. ${ }^{[25,26]} \mathrm{In}$ this sense, due to the MHD and magnetophoretic forces, we assume that the application of the magnetic field induces a more efficient penetration of the electrolyte, leading to the formation of oxide species and favouring the migration of $\mathrm{Fe}$ atoms from the inner part of the alloy particles to the surface, leaving behind fragments of almost unoxidized Ni clusters. Along this front, and corroborated by both ex-situ and in-situ STEM-EELS results, the magnetic field induces strong metal phase segregation, provoking the formation of crystalline nanometric ( $\leq$ $5 \mathrm{~nm}$ ) cluster-like nanoparticles of $\mathrm{Ni}$ in close contact, together with an enhanced oxidation of the nanoparticles surface. These processes explain the reduced saturation magnetization observed in the MNC-MC. Additionally, the phase segregation indicates a loss of nanoparticle bonding and coupling with the conducting graphene matrix, explaining the observed decrease of the $\mathrm{I}_{\mathrm{D}} / \mathrm{I}_{\mathrm{G}}$ ratio in the statistical Raman spectroscopy. ${ }^{[16]}$ The formation of these small clusters provides a dramatic increase of active sites for the development of Faradaic processes at the surface, in excellent accordance to the PEIS and XPS results, which suggest the presence of superficial oxide layers. Within the applied potential window, the very small Ni particle size and their $\mathrm{NiO}$ shell, which is a major source of pseudocapacitance ${ }^{[27]}$, creates efficient diffusion paths for the $\mathrm{OH}^{-}$ions, which significantly enhances the intercalation of the electrolyte ions and, therefore, the utilization of the electrode material, leading to an increase in the specific capacitance. Moreover, the resilient graphene matrix provides a conductive network between the metal clusters and acts as an optimal support avoiding sintering. This is the first time that the effect induced by a magnetic field results in a permanent improvement of the supercapacitive properties. This enhancement accounts for an impressive $1200 \%$, leading to specific capacitances values of more than $1800 \mathrm{~F} \cdot \mathrm{g}^{-1}$. This performance is very competitive taking into account the values of worldwide extended carbon-based commercial supercapacitors $\left(25-30 \mathrm{~F} \cdot \mathrm{g}^{-1}\right)$, as well as some of the most recent related publications $(<1010$ $\mathrm{F} \cdot \mathrm{g}^{-1}$ for NiO-carbon based supercapacitors in $\left.6 \mathrm{M} \mathrm{KOH}\right)^{[28]}$ and other carbon-based hybrid 
supercapacitors, e.g.: (950 F/g for $\mathrm{MnO}_{2}-\mathrm{CNT}$ hybrid supercapacitors ${ }^{[29]}$. Last but not least, we have checked the scope of this approach synthesizing an unprecedented NiCo-MNC analogue showing a similar behaviour with an increase of the specific capacitance of the device from 90 to $500 \mathrm{~F} \cdot \mathrm{g}^{-1}$; i.e. an enhancement of $~ 550 \%$ (Figure SI 27-32). These preliminary results point towards the universality of the magnetic-field-enhanced effect in hierarchical transition metal/carbon-based nanoscomposites.

In summary, the application of external magnetic fields to $\mathrm{FeNi}_{3}$-graphene nanocomposites during the galvanostatic charge-discharge cycles is twofold: On the one hand, it enhances the oxidation of the metal nanoparticles. On the other hand, it leads to an unprecedented metal phase segregation forming nanometric metal clusters of $\mathrm{Ni}$ in particular with an outstanding electrochemical activity. These newly formed metal clusters lead to a large increase of the $\mathrm{Ni} / \mathrm{NiO}$ interfaces, and accounts for the dramatic increase of the specific capacitance of the device (average values from 155 to $1840 \mathrm{~F} \cdot \mathrm{g}^{-1}$; i.e. an enhancement of $\sim 1200 \%$ ). These results open the door to an external magnetic field improvement of the specific capacitance in hybrid magnetic supercapacitors -a general feature taking into account that most of the conventionally employed faradaic materials consists of magnetic transition metals like $\mathrm{Ni}, \mathrm{Co}$ or $\mathrm{Fe}-$ and brings us closer to the development of promising new magnetically-switchable energy storage devices. Moreover, the intent of this work transcends energy storage because underlines the influence of a magnetic field during a electrochemical reaction in carbon-based nanocomposites.

\section{Supporting Information}

Supporting Information is available from the Wiley Online Library or from the author.

\section{Acknowledgements}


Financial support from the European Union (ERC Advanced Grant Mol-2D 788222, ERC Starting Grant 2D-PnictoChem 804110, ERC Proof of Concept Grant Hy-MAC 713704, ERC Proof of Concept Grant MAGTOOLS, and COST-Action on Molecular Spintronics (MOLSPIN CA15128)), the Spanish MINECO (Projects MAT2017-89943-R, RTI2018097895-B-C43 co-financed by FEDER, and the Unit of Excellence "Maria de Maeztu" MDM2015-0538), and the Generalitat Valenciana (Prometeo Program) is gratefully acknowledged. G.A. thanks for the support by the DFG (FLAG-ERA AB694/2-1) and the Generalitat Valenciana (CIDEGENT/2018/001 grant). G.A. received financial support through the Postdoctoral Junior Leader Fellowship Programme from "la Caixa" Banking Foundation. S.G.M. and J.R. thank the Spanish MINECO for their predoctoral grants. The authors thank Dr. Antonio Alberola-Catalan for his experimental support. Electron microscopy observations were carried out at the ICTS ELECMI node at Centro Nacional de Microscopía Electrónica at the Universidad Complutense de Madrid.

Received: ((will be filled in by the editorial staff))

Revised: ((will be filled in by the editorial staff)) Published online: ((will be filled in by the editorial staff)) 


\section{References}

[1] N. S. Lewis, D. G. Nocera, Proc. Natl. Acad. Sci. 2006, 103, 15729.

[2] H. B. Gray, Nat. Chem. 2009, 1, 7.

[3] V. Nicolosi, M. Chhowalla, M. G. Kanatzidis, M. S. Strano, J. N. Coleman, Science 2013, 340, 1226419.

[4] H. Wang, H. Dai, Chem. Soc. Rev. 2013, 42, 3088.

[5] F. Bonaccorso, L. Colombo, G. Yu, M. Stoller, V. Tozzini, A. C. Ferrari, R. S. Ruoff, V. Pellegrini, Science 2015, 347, 1246501.

[6] F. Beguin, E. Frackowiak, Supercapacitors: Materials, Systems and Applications, John Wiley \& Sons, 2013.

[7] G. Wang, L. Zhang, J. Zhang, Chem. Soc. Rev. 2012, 41, 797.

[8] R. N. O’Brien, ECS Trans. 2007, 3, 23.

[9] L. M. A. Monzon, J. M. D. Coey, Electrochem. Commun. 2014, 42, 38.

[10] L. M. A. Monzon, J. M. D. Coey, Electrochem. Commun. 2014, 42, 42.

[11] J. Zhu, M. Chen, H. Qu, Z. Luo, S. Wu, H. A. Colorado, S. Wei, Z. Guo, Energy Environ. Sci. 2012, 6, 194.

[12] J. Zhu, M. Chen, H. Wei, N. Yerra, N. Haldolaarachchige, Z. Luo, D. P. Young, T. C. Ho, S. Wei, Z. Guo, Nano Energy 2014, 6, 180.

[13] G. Abellán, E. Coronado, C. Martí-Gastaldo, A. Ribera, J. F. Sánchez-Royo, Chem. Sci. 2012, 3, 1481 .

[14] G. Abellán, E. Coronado, C. Martí-Gastaldo, A. Ribera, T. F. Otero, Part. Part. Syst. Charact. 2013, 30, 853 .

[15] G. Abellán, H. Prima-García, E. Coronado, J. Mater. Chem. C 2016, DOI 10.1039/C5TC04445F.

[16] H. Wang, Y. Liang, M. Gong, Y. Li, W. Chang, T. Mefford, J. Zhou, J. Wang, T. Regier, F. Wei, H. Dai, Nat. Commun. 2012, 3, 917. 
[17] G. Abellán, M. Schirowski, K. F. Edelthalhammer, M. Fickert, K. Werbach, H. Peterlik, F. Hauke, A. Hirsch, J. Am. Chem. Soc. 2017, 139, 5175.

[18] C. h. Bajorek, M. Nicolet, C. h. Wilts, Appl. Phys. Lett. 1971, 19, 82.

[19] K. Asami, K. Hashimoto, Corros. Sci. 1984, 24, 83.

[20] C. Colliex, T. Manoubi, C. Ortiz, Phys. Rev. B 1991, 44, 11402.

[21] S. R. Ragsdale, K. M. Grant, H. S. White, J. Am. Chem. Soc. 1998, 120, 13461.

[22] T. Z. Fahidy, J Apple Electrochem 1983, 13, 553.

[23] R. J. Moreau, Magnetohydrodynamics, Kluwer Academic Publishers, Norwell, MA, USA, 1990.

[24] L. Pietrobon, L. Fallarino, A. Berger, A. Chuvilin, F. Casanova, L. E. Hueso, Small 2015, 11, 6295 .

[25] Y. Lu, T. Zhu, G. Zhang, Z. He, C. Lin, Y. Chen, H. Guo, RSC Adv. 2015, 5, 99745.

[26] M. Wang, Q. Chen, Chem. - Eur. J. 2010, 16, 12088.

[27] Q. Lu, M. W. Lattanzi, Y. Chen, X. Kou, W. Li, X. Fan, K. M. Unruh, J. G. Chen, J. Q. Xiao, Angew. Chem. Int. Ed. 2011, 50, 6847.

[28] J. Cheng, B. Zhao, W. Zhang, F. Shi, G. Zheng, D. Zhang, J. Yang, Adv. Funcinal Mater. 2015, 25, 7381.

[29] J. Yan, Z. Fan, T. Wei, J. Cheng, B. Shao, K. Wang, L. Song, M. Zhang, J. Power Sources 2009, 194, 1202. 
a

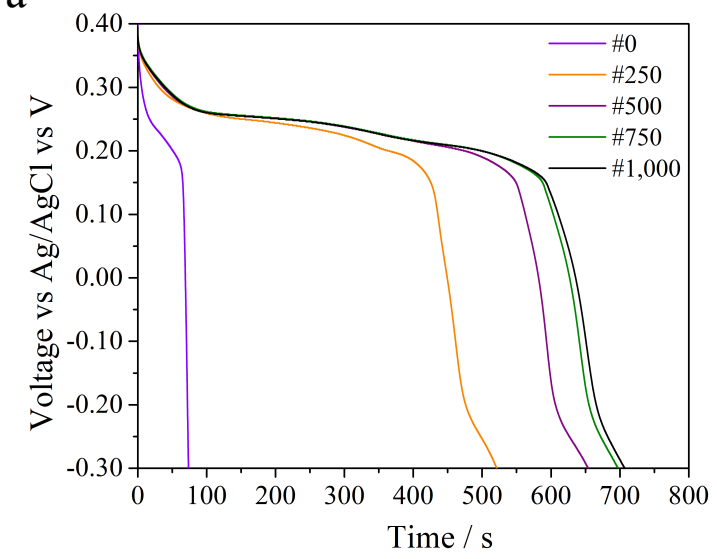

b

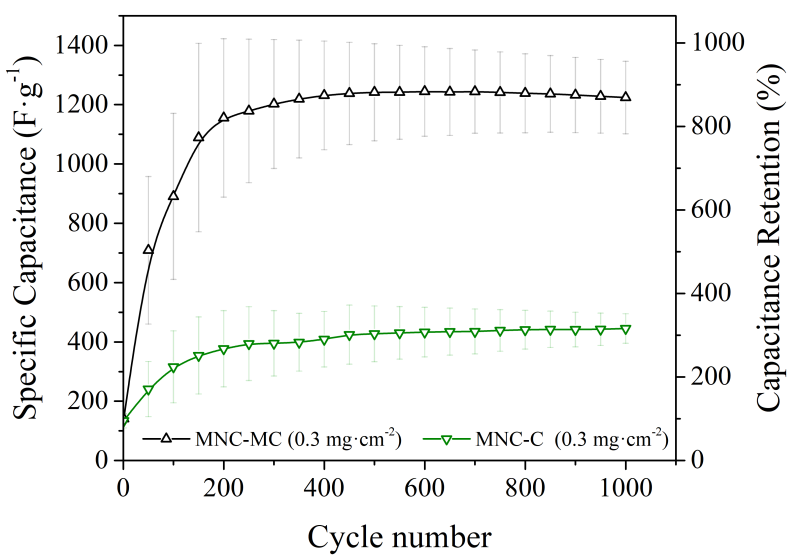

Figure 1. Electrochemical measurements under applied magnetic field. a) Galvanostatic discharges of the material in the presence of an external magnetic field showing the increase of the discharge-time with the increase in the cycle number. b) Comparative capacitance retention of the material over 1,000 cycles with (MNC-MC) and without (MNC-C) an external applied magnetic field and their corresponding specific capacitance values. 
a

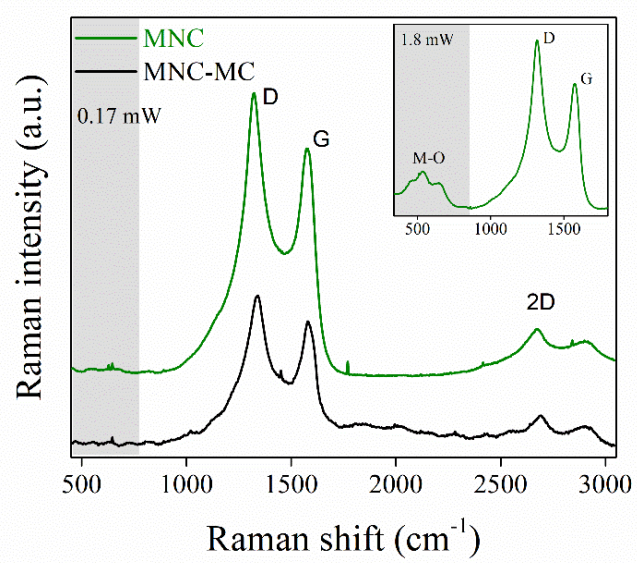

C

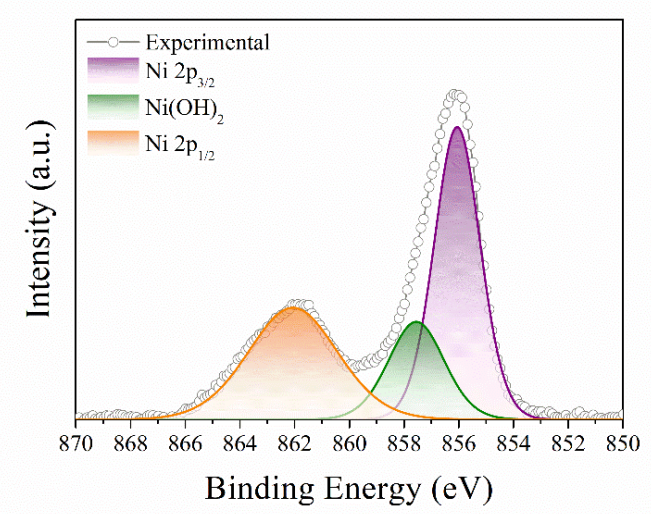

b

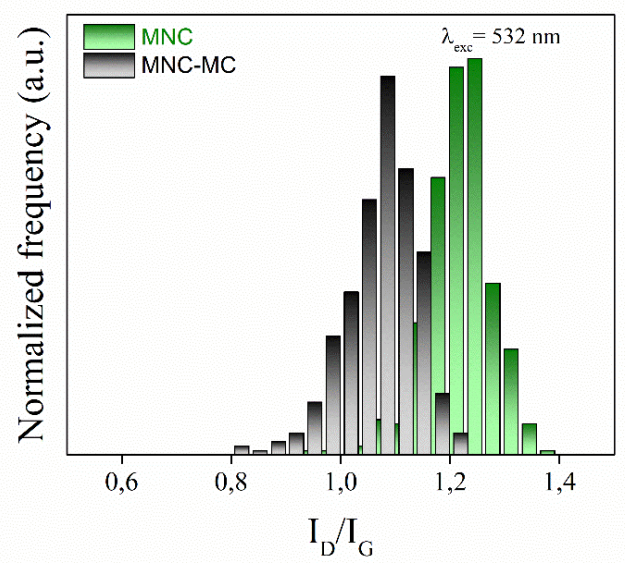

d

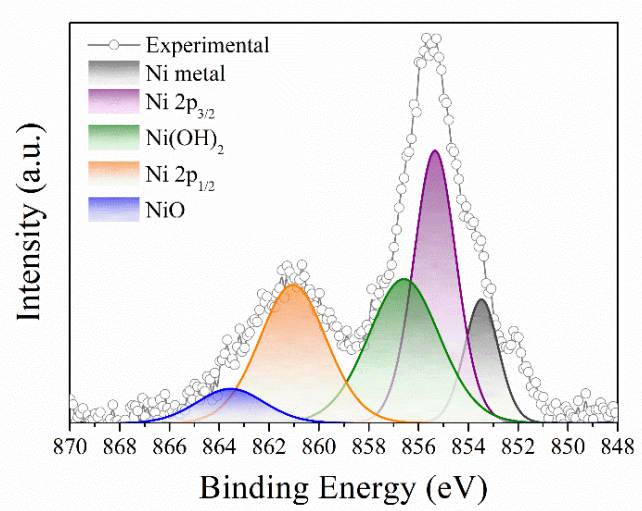

Figure 2. Raman, XPS and magnetic characterization of MNC and MNC-MC. a) Statistical Raman spectroscopy of MNC (green) and MNC-MC (black) showing the average spectra collected at $0.17 \mathrm{~mW}\left(\lambda_{\text {exc }}=532 \mathrm{~nm}\right)$. The inset highlights the influence of laser power unveiling characteristic peaks associated to $\mathrm{M}-\mathrm{O}$ vibrations, corresponding to the encapsulated $\mathrm{FeNi}_{3}$ nanoparticles. b) Raman histograms ( $\mathrm{I}_{\mathrm{D}} / \mathrm{I}_{\mathrm{G}}$ ratio distribution) of the $\mathrm{MNC}$ and MNC-MC, showing the decrease in the $\mathrm{I}_{\mathrm{D}} / \mathrm{I}_{\mathrm{G}}$ after the application of the magnetic field. $\mathrm{c}$ ) High resolution XPS $\mathrm{Ni}_{2 \mathrm{p}}$ spectra of $\mathrm{MNC}$ and d) MNC-MC confirm that nickel oxidation is favored in presence of the magnetic field. 

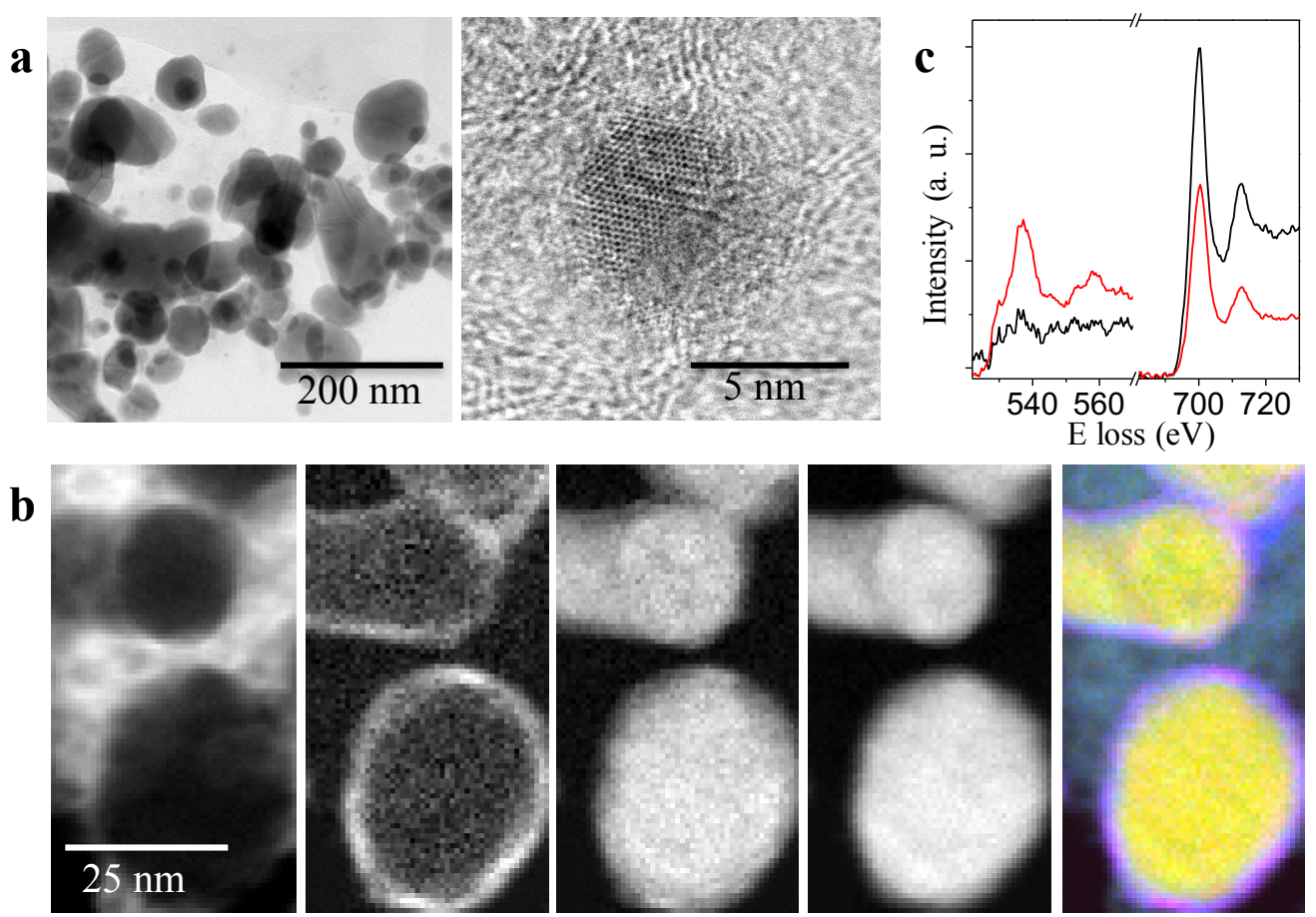

$\mathrm{C}=\mathrm{C}$

$\mathrm{B}=\mathrm{O}$

$\mathrm{R}=\mathrm{Fe}$

$\mathrm{G}=\mathrm{Ni}$

\section{$\mathrm{C} K$}

$\mathrm{O} K$

Fe $L_{2,3}$

Ni $L_{2,3}$

Figure 3. STEM and EELS analysis of MNC. a) Low (left) and high (right) resolution ABF images of the pristine MNC sample, obtained at $200 \mathrm{kV}$. b) Compositional maps, obtained via multiple linear least squares fitting of raw EELS data, corresponding to the $\mathrm{C} \mathrm{K}, \mathrm{O} \mathrm{K}, \mathrm{Fe} \mathrm{L}_{2,3}$ and $\mathrm{Ni} \mathrm{L}_{2,3}$ edges. The left panel shows a false overlay of the former, corresponding cyan $(\mathrm{C})$, blue (B), red (R) and green (G) for C, O, Fe and Ni, respectively. c) Averaged EEL spectra obtained from the surface (red) and the center of a pristine MNC nanoparticle. EELS data acquired at $80 \mathrm{kV}$. 


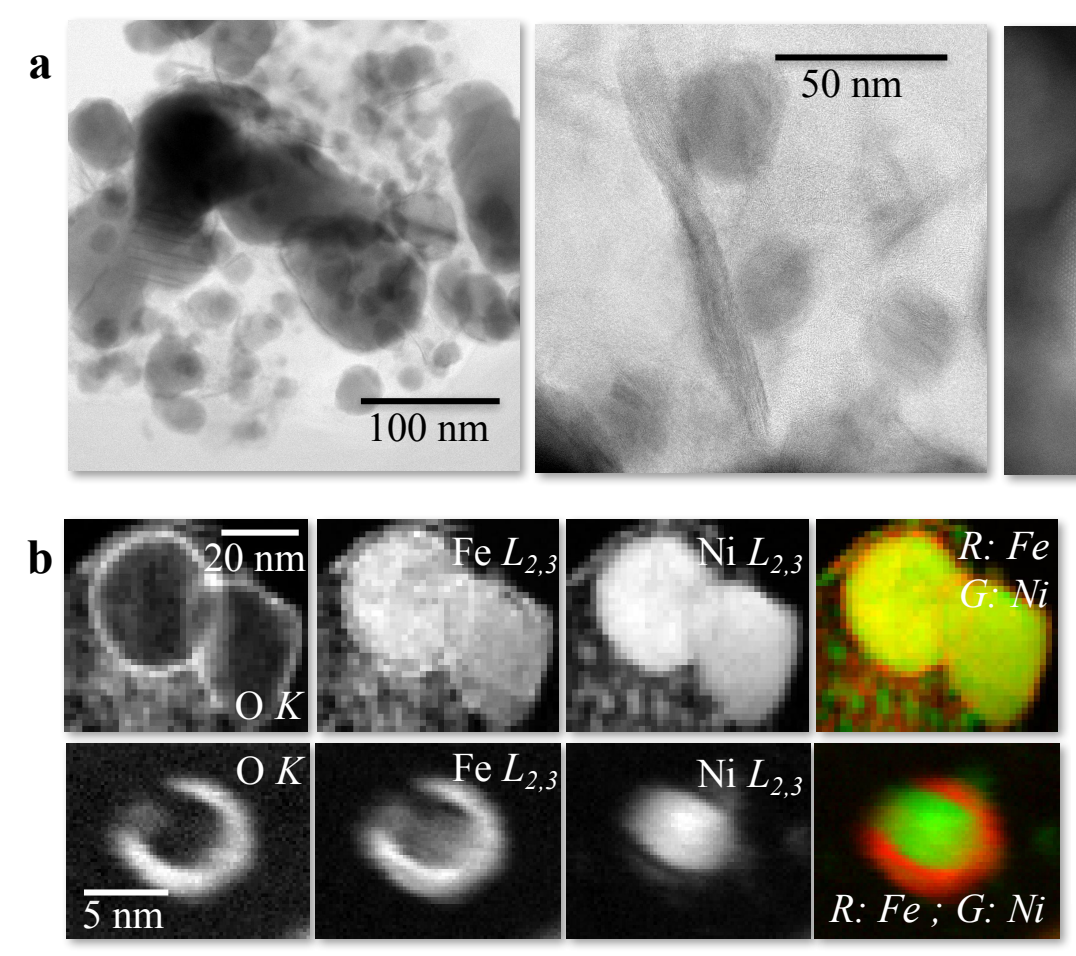

Figure 4. STEM and EELS analysis of MNC-MC. a) Low and intermediate magnification

ABF images, along with a high resolution HAADF image of the MNC-MC specimen, acquired at $200 \mathrm{kV} . \mathrm{b}$ ) Intermediate and high magnification EELS maps, including the O K, $\mathrm{Fe} \mathrm{L}_{2,3}$ and $\mathrm{Ni} \mathrm{L}_{2,3}$ maps. Color maps show an overlay of the respective $\mathrm{Fe}$ (red) and $\mathrm{Ni}$ (green) maps. c) EEL spectrum from the Fe oxide surface of a cluster. EELS data recorded at $80 \mathrm{kV}$. 
$\mathbf{a}$
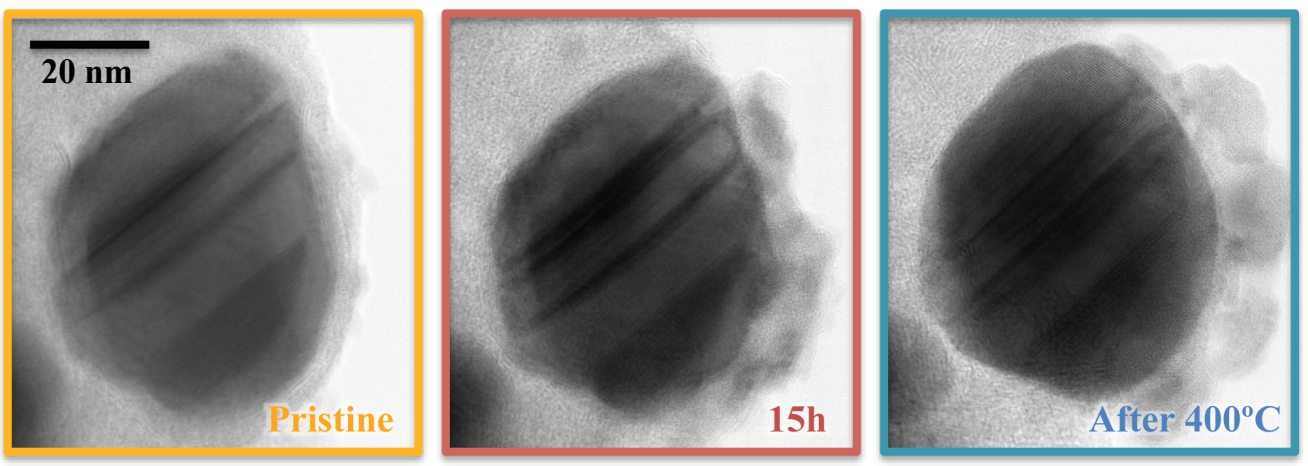

b
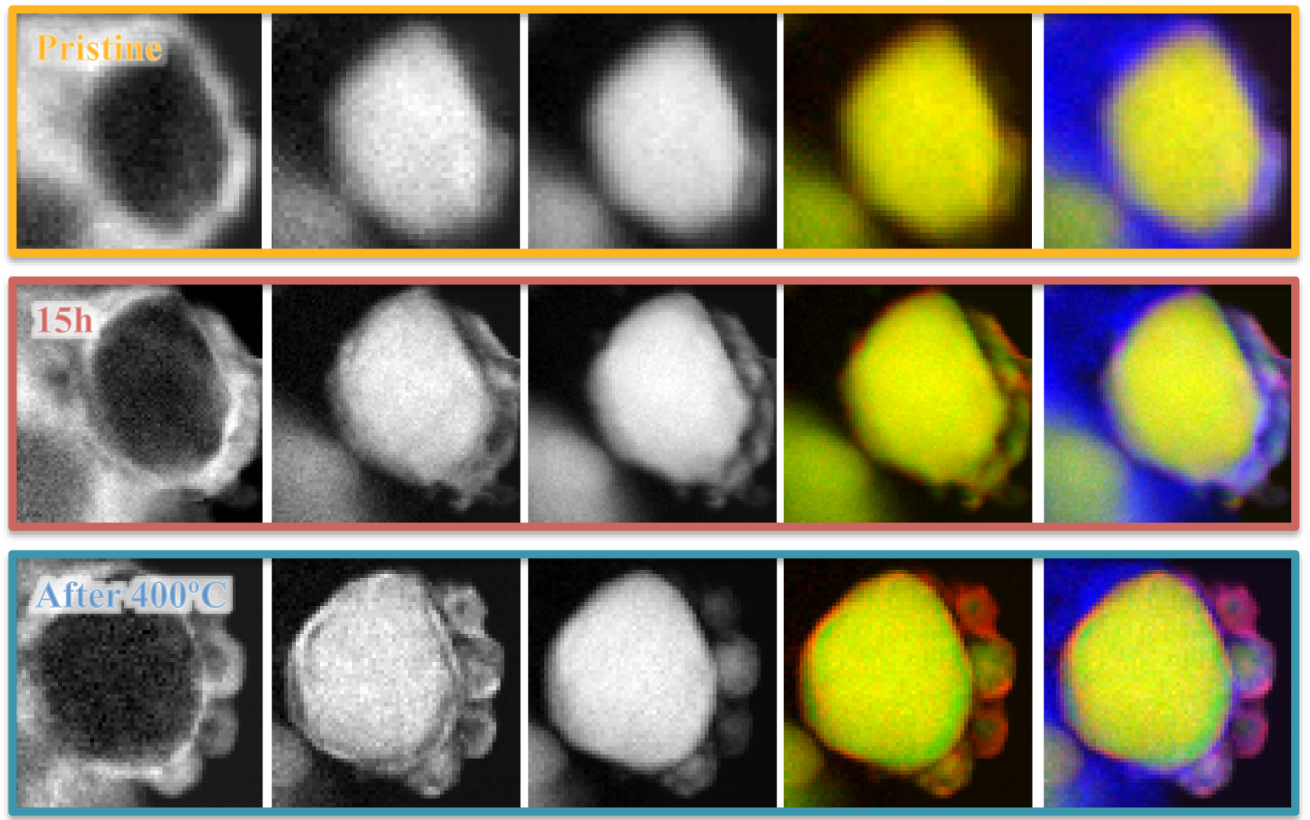

$\operatorname{Fe} L_{2,3}$

Ni $L_{2,3}$

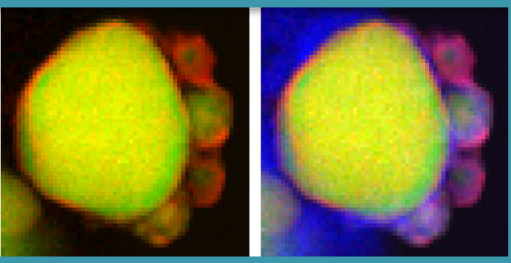

$\mathrm{Fe}=$ red, $\mathrm{Ni}=$ green, $\mathrm{O}=$ blue

Figure 5. STEM-EELS analysis after in-situ treatments. a) Sequential atomic resolution ABF images of a MNC particle. From left to right: pristine sample, after 15 hours in the objective lens magnetic field (>2T) and after a 30 minutes annealing at $400^{\circ} \mathrm{C}$ and fast quench back to RT, always under applied magnetic field. The scale bar represents $10 \mathrm{~nm}$. b) EELS maps of the same particle. From left to right: $\mathrm{O} \mathrm{K}, \mathrm{Fe}_{2,3}$, Ni $\mathrm{L}_{2,3}$, along with colour overlays where red represents the Fe map, green is $\mathrm{Ni}$ and blue is $\mathrm{O}$. From top to bottom: pristine sample, after 15 hours in the objective lens magnetic field $(>2 \mathrm{~T})$ and after a 30 minutes annealing at $400^{\circ} \mathrm{C}$ and fast quench back to RT, always under applied magnetic field. Some spatial drift during EELS mapping is present. All data acquired at room temperature at $80 \mathrm{kV}$. 